\title{
Hybrid therapy as first-line regimen for Helicobacter pylori eradication in a high clarithromycin resistance area: a prospective open-label trial
}

\author{
Sotirios D. Georgopoulos ${ }^{a}$, Vasilios Papastergiou ${ }^{b}, B^{2}$ atriz Martinez-Gonzalez ${ }^{c}$, Elias Xirouchakis ${ }^{a}$, \\ Ioannis Familias ${ }^{b}$, Dionysis Sgouras ${ }^{c}$, Andreas Mentisc, Stylianos Karatapanis ${ }^{b}$
}

Athens Medical, Paleo Faliron Hospital, Athens; General Hospital of Rhodes; Hellenic Pasteur Institute, Athens, Greece

\section{Abstract}

${ }^{\mathrm{a} G a s t r o e n t e r o l o g y ~ D e p a r t m e n t, ~ A t h e n s ~ M e d i c a l, ~ P a l e o ~ F a l i r o n ~}$ Hospital, Athens (Sotirios D. Georgopoulos, Elias Xirouchakis); ${ }^{\text {FFirst }}$ Department of Internal Medicine, General Hospital of Rhodes, Rhodes (Vasilios Papastergiou, Ioannis Familias, Stylianos Karatapanis); 'Laboratory of Medical Microbiology, Hellenic Pasteur Institute, Athens (Beatriz Martinez-Gonzalez, Dionysis Sgouras, Andreas Mentis), Greece

Conflict of interest: None

Correspondence to: Sotirios D. Georgopoulos, MD, FEBGH, AGAF, Director, Gastroenterology and Hepatology Department, Athens Medical, Paleo Faliron General Hospital, Athens, Greece, 36 Areos str., 17462, Paleo Faliron, Greece, e-mail: georgpap@ath.forthnet.gr

Received 24 September 2017; accepted 26 October 2017; published online 15 December 2017

DOI: https://doi.org/10.20524/aog.2017.0221

\section{Introduction}

As a result of rising antibiotic resistance, the eradication rate of Helicobacter pylori (H. pylori) using standard triple therapies is unacceptably low $(<80 \%)$ in most countries [1]. Therefore, bismuth-based and non-bismuth quadruple first-line options have been proposed, aiming to overcome increasing clarithromycin resistance [2,3]. A rational approach is to use a clarithromycin-free regimen (i.e., bismuth-based quadruple therapy). Nevertheless, non-bismuth quadruple therapies are the only realistic approach in countries where bismuth salts and/or tetracycline are not available [4]. An example is Greece, a country with high prevalence of resistance to clarithromycin (>27\%) and metronidazole ( $>40 \%$ ) [5-7]. Until recently, the sequential and concomitant regimens have been the competing 
non-bismuth quadruple treatments, with several studies assessing their efficacy, safety, and compliance rates. Finally, the 2015 Maastricht V/Florence consensus favored the use of concomitant (over sequential) therapy, by virtue of its better efficacy against antibiotic-resistant $H$. pylori strains supported by head-to-head trials $[8,9]$.

Since 2011, the armamentarium of non-bismuth quadruple treatments has further expanded. This was due to the addition of an innovative 14-day, two-step regimen that functionally combines the concomitant and sequential treatments (i.e., hybrid therapy) [10]. It comprises a proton pump inhibitor (PPI) and amoxicillin for 14 days, adding clarithromycin and a nitroimidazole as a quadruple therapy for the final 7 days $[2,10]$. Compared to a concomitant treatment of same duration (14 days), the hybrid regimen is characterized by a lower pill burden. This could be associated with potential advantages, including a lower cost of eradication but also improved safety, convenience and better compliance. In the original pilot study, hybrid therapy produced outstanding cure rates, yielding 97\% in intention to treat (ITT) and 99\% in per protocol (PP) analysis [10]. However, this study was conducted in a setting (Taiwan) with low clarithromycin resistance. Thus, further validation is warranted for hybrid therapy, providing concrete evidence of its efficacy in geographical and/or national areas harboring different patterns of antimicrobial resistance [11]. To date, only a limited number of studies have evaluated hybrid therapy in a high-resistance setting, and even fewer provided pretreatment susceptibility data. Therefore, this prospective open-label trial was aimed to determine the clinical efficacy and safety of the hybrid regimen in treatment-naïve patients in Greece. The impact of $H$. pylori resistance to clarithromycin and metronidazole on the efficacy of hybrid therapy was also evaluated.

\section{Patients and methods}

\section{Study design and participants}

This was a prospective, open-label, dual-center study for the treatment of patients with $H$. pylori infection. The study was conducted from January 2015 to March 2016 in the Gastroenterology Departments of the Athens Medical, Paleo Faliron General Hospital (Athens, Greece) and the General Hospital of Rhodes (Rhodes, Greece). Consecutive patients with dyspepsia who were referred and scheduled for upper endoscopy were prospectively enrolled. Eligible patients were those aged 18 years, diagnosed with $H$. pylori infection by rapid urease test confirmed with gastric histology and/or culture. The following were exclusion criteria: a history of previous $H$. pylori eradication treatment; allergies to the medications used; treatment during the preceding two months with antibiotics, bismuth preparations or non-steroid anti-inflammatory drugs, and in the preceding two weeks with PPIs; previous esophageal or gastric surgery; serious systemic disease; pregnancy or lactation. For eligible patients, the demographic data, body mass index (BMI), history of smoking and alcohol consumption, and the endoscopic findings were all recorded.

The study protocol was in accordance with the principles of the Declaration of Helsinki and was approved by the local Ethics Committee of the participating hospitals. Written informed consent was obtained from all patients prior to their enrollment in the study.

\section{Treatment and procedures}

All eligible patients were assigned to receive a hybrid regimen consisting of $40 \mathrm{mg}$ esomeprazole and $1 \mathrm{~g}$ amoxicillin for 14 days, with the addition of $500 \mathrm{mg}$ clarithromycin and $500 \mathrm{mg}$ metronidazole for the final 7 days, all twice daily. A ${ }^{13} \mathrm{C}$-urea breath test (Helicobacter Test INFAI ${ }^{\oplus}$, INFAI $\mathrm{GmbH}$, Cologne, Germany) was performed 8-12 weeks after completion of treatment and a negative test result was considered indicative of successful $H$. pylori eradication. For patients who required a follow-up endoscopy because of gastric ulcer, histological evaluation of gastric biopsies (two from the corpus and two from the antrum) was the diagnostic test.

Treatment-related side-effects were investigated by means of a structured questionnaire, immediately after therapy was completed. Patients were asked to grade the severity of each side-effect as follows: a) mild: transient and well tolerated; b) moderate: causing discomfort and partially interfering with common everyday activities; c) severe: causing considerable interference with the patient's activities, possibly incapacitating or life-threatening. Drug compliance was determined by counting unused medication. For this purpose, any tablet that was not consumed was brought back to the clinic for pill count. Patients' adherence to therapy was defined as poor if $<90 \%$ of the prescribed medication was taken. Biopsy specimens were cultured according to previously described methods and antibiotic susceptibility was determined by Etest ${ }^{\circledast}$ (AB Biodisk, Solna, Sweden) [5,7]. H. pylori strains with MICs of $>1 \mu \mathrm{g} / \mathrm{mL}$, $>0.5 \mu \mathrm{g} / \mathrm{mL}$ and $>8 \mu \mathrm{g} / \mathrm{mL}$ were considered to be resistant to clarithromycin, amoxicillin and metronidazole, respectively. Culture and antibiotic susceptibility testing procedures were centralized and performed by blinded personnel at the Laboratory of Medical Microbiology of the Hellenic Pasteur Institute (Athens, Greece).

\section{Statistical analysis}

The primary outcome was eradication of $H$. pylori infection. In the ITT analysis, data from all included patients were calculated and patients who did not complete the study were counted as treatment failures. PP analysis excluded patients who violated the study protocol or who were noncompliant with the prescribed treatment. Secondary outcomes were compliance and side effects.

Categorical variables are expressed as percentages, while continuous variables are expressed as means with standard deviation. The chi-square test or the Fisher's exact test were used 
to compare categorical data, as appropriate. The eradication rates and their $95 \%$ confidence intervals (CI) were calculated.

Univariate analysis was performed to evaluate factors affecting the eradication efficacy of hybrid therapy. A multivariate regression analysis was planned, including variables with statistical significance $(\mathrm{P}<0.1)$ on univariate analysis. The magnitude of the effect is described as odds ratio (OR) with 95\%CI. SPSS version 24 for Macintosh (IBM SPSS, Chicago, IL, USA) was used for statistical analyses and a twosided P-value of $<0.05$ was regarded as statistically significant.

\section{Results}

\section{Patient data and antibiotic resistance rates}

The study flow chart is shown in Fig. 1. A total of 343 patients were initially screened for eligibility and 188 were excluded. Thus, 155 patients were finally included in the study; their baseline characteristics are shown in Table 1. H. pylori culture and sensitivity test were successfully carried

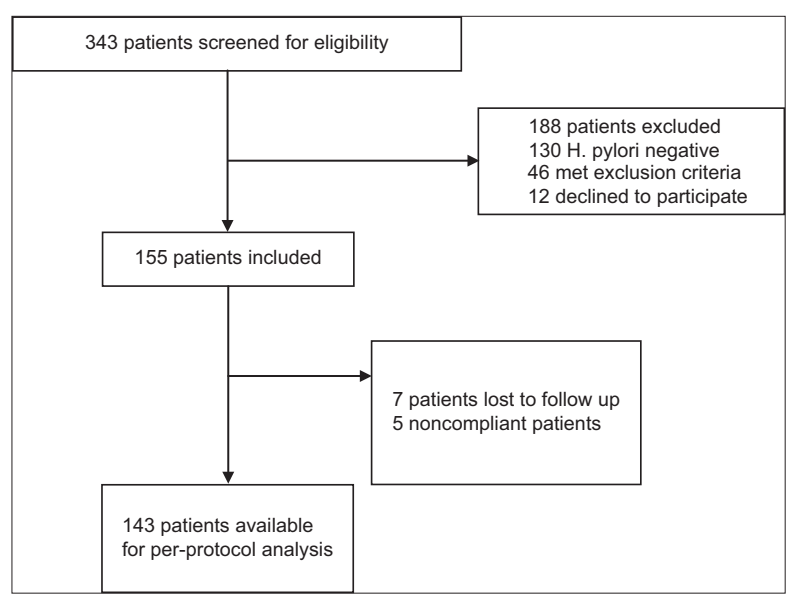

Figure 1 Flowchart of the patients in the study H. pylori, Helicobacter pylori

Table 1 Clinical and demographic characteristics of the treated population

\begin{tabular}{lc}
\hline Characteristics & $\mathrm{n}=155$ \\
\hline Age, mean (SD) & $50.5(12.4)$ \\
Male sex, n (\%) & $90(58.1)$ \\
BMI, mean (SD) & $25.6(3.7)$ \\
Alcohol, n (\%) & $21(13.5)$ \\
Smokers, n (\%) & $50(32.3)$ \\
DUD, n (\%) & $30(19.4)$ \\
GUD, n (\%) & $6(3.9)$ \\
\hline NUD, n (\%) & $119(76.8)$ \\
\hline
\end{tabular}

SD, standard deviation; $B M I$, body mass index; DUD, duodenal ulcer disease; GUD, gastric ulcer disease; NUD, non-ulcer dyspepsia out in $135 / 155(87.1 \%)$ patients. The rates of $H$. pylori strains with single resistance to clarithromycin, metronidazole and amoxicillin were 35/135 (25.9\%), 42/135 (31.1\%) and 0/135 (0\%), respectively, whereas dual clarithromycin/ metronidazole resistance was detected in 12/135 (8.9\%).

\section{Side effects and adherence}

One hundred fifty-five patients were included in the trial, but a total of seven patients were lost to follow up (Fig. 1). Among the 148 patients who returned for follow up, a total of 58 adverse events were noted in $44(29.7 \%)$ patients. The proportion and severity of adverse events is shown in Table 2. Overall, 2 of the 148 patients (1.3\%) specified severe adverse events as the reason for treatment interruption. One patient discontinued treatment on day 11 because of abdominal discomfort; however, $H$. pylori was successfully eradicated, as indicated by the negative breath test on final follow up. However, the other patient, who interrupted treatment on day 3 because of an allergic skin rash, failed to eradicate the pathogen. Overall, 96.6\% (143/148) of the patients took more than $90 \%$ of the total medication prescribed. Adherence to therapy, defined as the total percentage of tablets taken, was 98.3\% (95\%CI 98.1-98.5\%).

\section{Eradication efficacy and associated factors}

Overall, eradication rates were $85.8 \%(133 / 155 ; 95 \%$ CI 79.4 90.5\%) and 90.2\% (129/143; 95\%CI 84.1-94.2\%) respectively, in the ITT and PP analyses. No differences in the eradication rates were found between the clarithromycin-resistant and -sensitive strains $(91.4 \%$ [32/35] vs. $94.3 \%$ [83/88], $\mathrm{P}=0.69)$, or between the metronidazole-resistant and -sensitive strains (90.5\% [38/42] vs. 95.1\% [77/81], P=0.44). However, dual clarithromycin-metronidazole resistance was associated with a significantly lower eradication rate $(50 \%$ [6/12]) compared with single clarithromycin resistance (91.4\% [32/35], $\mathrm{P}=0.005)$, single metronidazole resistance $(90.5 \%$ [38/42], $\mathrm{P}=0.005)$ and dual susceptibility $(97.8 \%$ [45/46], $\mathrm{P}=0.0001)$. By univariate analysis (Table 3 ), the following variables were not associated with $H$. pylori eradication: sex, age, BMI, history of smoking and alcohol consumption, indication for eradication (peptic ulcer disease vs. non-ulcer dyspepsia), study center, and single clarithromycin or metronidazole resistance. The presence of dual clarithromycin/metronidazole resistance was the only factor associated with the failure of hybrid therapy (OR 14.4, 95\%CI 3.8-54.9, $\mathrm{P}=0.0003$ ).

\section{Discussion}

Six years after it was first proposed, our understanding of hybrid therapy remains limited. Available evidence from 12 studies (2009-2015) has indeed shown a wide variation in 
Table 2 Adverse events resulting from eradication therapy

\begin{tabular}{lcccc}
\hline Side effects & Mild (n) & Moderate (n) & Severe (n) & Total (n, \%) \\
\hline Total & 42 & 14 & 2 & 1 \\
Abdominal discomfort & 10 & 3 & 0 & $14(24.1)$ \\
Nausea & 7 & 2 & 0 & $9(15.5)$ \\
Taste disturbance & 12 & 4 & 0 & $16(27.6)$ \\
Vomiting & 1 & 1 & 0 & $2(3.4)$ \\
Diarrhea & 7 & 1 & 0 & $8(13.8)$ \\
Headache & 3 & 0 & 1 & $3(5.2)$ \\
Skin rash & 0 & 0 & 0 & $1(1.7)$ \\
Other & 2 & 3 & $5(8.6)$ \\
\hline
\end{tabular}

Table 3 Factors affecting the eradication efficacy (per protocol) of hybrid therapy as determined by univariate analysis

\begin{tabular}{|c|c|c|}
\hline Factors & $\begin{array}{l}\text { Eradication } \\
\text { efficacy } \\
\mathrm{n} / \mathrm{N}(\%)\end{array}$ & P-value \\
\hline \multicolumn{3}{|l|}{ Sex } \\
\hline Male & $74 / 82(90.2)$ & \multirow[t]{2}{*}{1.00} \\
\hline Female & $55 / 61(90.2)$ & \\
\hline \multicolumn{3}{|c|}{ Age (years) } \\
\hline$<46$ & $59 / 64(92.2)$ & \multirow[t]{2}{*}{0.58} \\
\hline$\geq 46$ & $70 / 79(88.6)$ & \\
\hline \multicolumn{3}{|l|}{ Smoking } \\
\hline Yes & $43 / 50(86)$ & \multirow[t]{2}{*}{0.25} \\
\hline No & $86 / 93(92.5)$ & \\
\hline \multicolumn{3}{|l|}{ Alcohol } \\
\hline Yes & $15 / 17(88.2)$ & \multirow{2}{*}{0.67} \\
\hline No & $114 / 126(90.5)$ & \\
\hline \multicolumn{3}{|l|}{ Diagnosis } \\
\hline PUD & 34/36 (94.4) & \multirow{2}{*}{0.52} \\
\hline NUD & $95 / 107(88.8)$ & \\
\hline \multicolumn{3}{|c|}{ BMI $\left(\mathrm{kg} / \mathrm{m}^{2}\right)$} \\
\hline$<25$ & $71 / 77(92.2)$ & \multirow[t]{3}{*}{0.40} \\
\hline $25-29$ & $42 / 47(89.4)$ & \\
\hline$\geq 30$ & $16 / 19(84.2)$ & \\
\hline \multicolumn{3}{|c|}{ Study center } \\
\hline Site A & $84 / 92(91.3)$ & \multirow[t]{2}{*}{0.57} \\
\hline Site B & $45 / 51(88.2)$ & \\
\hline \multicolumn{3}{|c|}{ Single clarithromycin resistance } \\
\hline Absent & $83 / 88(94.3)$ & \multirow[t]{2}{*}{0.69} \\
\hline Present & $32 / 35(91.4)$ & \\
\hline \multicolumn{3}{|c|}{ Single metronidazole resistance } \\
\hline Absent & 77/81 (95.1) & \multirow{2}{*}{0.44} \\
\hline Present & $38 / 42(90.5)$ & \\
\hline \multicolumn{3}{|c|}{$\begin{array}{l}\text { Dual clarithromycin/ } \\
\text { metronidazole resistance }\end{array}$} \\
\hline Absent & $115 / 123(93.5)$ & \multirow[t]{2}{*}{0.0003} \\
\hline Present & $6 / 12(50)$ & \\
\hline
\end{tabular}

the eradication rates, ranging from $77.6-97.4 \%$ in ITT and from $82.6-99.1 \%$ in PP analysis [12]. These heterogeneous results underscore the need for cautious interpretation of data pertaining to different regions and populations, which are likely to reflect different backgrounds of antibiotic resistance. To date, only a few studies evaluating hybrid therapy provided susceptibility data, mostly from regions with lowto-moderate clarithromycin resistance $[10,13]$. Moreover, the subpopulations tested were inherently small, thus raising the possibility of selection bias. This prospective trial, involving 155 patients from two hospitals located in two geographically distinct areas of Greece, indicates that hybrid therapy is a valuable (PP eradication: 90.2\%) treatment option that is safe and well-tolerated by most patients. This was achieved in the face of high $(>15 \%)$ clarithromycin, but acceptable $(<9 \%)$ dual clarithromycin/metronidazole resistance, documented by extensive analysis of susceptibility data $(n=135)$ [8]. To our knowledge, this is the first evaluation of hybrid therapy in Greece and represents the largest European series of antibioticresistant $H$. pylori strains treated with hybrid therapy.

We determined that the efficacy of hybrid therapy is markedly decreased by dual clarithromycin/metronidazole resistance. This premise has been also corroborated in previous studies that evaluated sequential and concomitant regimens $[5,7,14,15]$. Therefore, all non-bismuth quadruple therapies appear to be influenced by dual resistance, though not to the same extent. Rates of dual resistance $>5 \%,>9 \%$ and $>15 \%$ have been proposed as the thresholds associated with unacceptable performance ( $<90 \%$ cure rate) for sequential, hybrid and concomitant regimens, respectively [16]. Overall, our experience appears to support this notion, indicating that both the 10-day concomitant and 14-day hybrid therapies, but not the sequential therapy, are acceptable first-line regimens in Greece, a country with a $>5 \%$ but $<9 \%$ prevalence of dual clarithromycin/metronidazole resistance $[5,7,17,18]$. A head-to-head comparison between the 10-day concomitant and 14-day hybrid regimens would be useful to clarify the optimal first-line regimen in Greece. However, assuming they prove to be equally effective, the lower pill burden (80 tablets for 10-day concomitant; 84 tablets for 14-day hybrid) and less complex administration would favor the 10-day concomitant therapy. Crucially, shortening the duration of hybrid therapy (to 10 or 12 days) may be realistic in regions of moderate-to-low resistance [13]. However, this may be unfeasible in high-resistance settings, as shown by an 
Iranian randomized study comparing 14- and 10-day hybrid therapies (PP eradication: $92.8 \%$ and $83.8 \%$ respectively) [19]. Likewise, prolonged (14 days) exposure to amoxicillin is the key for improved eradication with hybrid therapy. Accordingly, prolonging the duration of sequential therapy (in which amoxicillin is discontinued at mid-point) does not seem to provide any substantial benefit [20]. To date, a single study from China recorded such a large dataset $(n=135)$ of antibiotic susceptibility before hybrid therapy [21]. In sharp contrast to our data, hybrid therapy was shown to perform unsatisfactorily (ITT: 77\%, PP: 86\%), an unsurprising finding considering that the burden of antibiotic resistance was disproportionately higher (clarithromycin 44.9\%, metronidazole 67.3\%, dual 33.3\%). Interestingly, the deleterious impact of dual resistance appears to be more prominent in the European as compared to the Asian population (efficacy against dual-resistant $H$. pylori strains: $70.2 \%$ in the Chinese study vs. $50 \%$ in present series) [21]. In keeping with this observation, only one out of three dual-resistant H. pylori strains was successfully eradicated in a Spanish/Italian study [22]. Clearly, a number of potential confounders may play a role, including racial and genetic background, differences in the metabolism of PPIs, and the nature of the underlying disease. Furthermore, variations in the type of PPI and nitroimidazole may affect the eradication outcome. Notably, tinidazole, which is known to possess a longer half-life compared to metronidazole, was used in the Chinese study. With regard to acid suppression, we used high-dose ( $40 \mathrm{mg}$ b.i.d.) esomeprazole, a new-generation PPI that has been reported to be metabolized irrespective of the cytochrome P450 (CYP) 2C19 status [23]. In contrast, standarddose (20 mg b.i.d.) omeprazole was used in the Chinese study, justified by the lower prevalence of rapid metabolizers among the Asian population [24]. Clearly, both studies address the same effect (i.e., detrimental impact of dual resistance on hybrid therapy). However, they addressed different settings of antibiotic resistance and distinct populations that were likely to reflect different magnitudes of this same phenomenon. Thus, rather than contrasting, the two studies should be viewed as complementary.

As sequential therapy, hybrid therapy has been devised to decrease pill burden (compared to concomitant therapy) with no efficacy cost, relying on sequential administration. It has been postulated that pretreatment with amoxicillin could markedly reduce the bacterial load and prevent the development of bacterial efflux channels [25], increasing the susceptibility of the organisms during the second phase of treatment [26]. Nevertheless, the concept of sequential administration has been seriously challenged by studies showing that "reverse hybrid" and "reverse sequential" regimens perform equally well [27,28]. Indeed, in a recent Taiwanese trial, reverse hybrid therapy was highly effective ( $95.7 \%$ by PP analysis) and superior to standard triple therapy [27].

In keeping with our findings, previous studies evaluating hybrid therapy reported consistently good compliance and safety [10,13,19,29-33]. A Spanish/Italian study determined that the overall rate of adverse effects of hybrid therapy was lower than that of concomitant therapy [22]. Likewise, the rate of discontinued medication was lower in a Korean study [31]. However, in both studies the differences were of borderline significance $(\mathrm{P}=0.05$ and $\mathrm{P}=0.051$, respectively). Overall, based on updated meta-analyses, hybrid therapy appears to display slightly better compliance and safety than concomitant therapy [12,34]. However, whether this difference may be clinically relevant, particularly regarding a routine practice setting, merits further assessment.

The present trial is not free of limitations. Firstly, by design, this was an open-label, non-controlled study, precluding conclusions on the comparative efficacy and safety of hybrid therapy. Secondly, the antimicrobial susceptibility of $H$. pylori was determined using the E-test, which tends to overestimate metronidazole resistance, although in a previous study this was not associated with any change in the overall metronidazole resistance pattern [7]. Thirdly, CYP2C19 polymorphisms were not evaluated. However, we expect the impact, if any, to be minimal, as the metabolism of esomeprazole has been reported to be independent of CYP2C19 status [35].

In conclusion, hybrid therapy achieved an $H$. pylori cure rate of $90.2 \%(P P)$ in a setting with high resistance to clarithromycin and metronidazole. Dual clarithromycin and metronidazole resistance plays a key role in the treatment failure of hybrid therapy. Further studies providing susceptibility data are warranted to better define the position of hybrid therapy in the armamentarium of current first-line regimens for $H$. pylori eradication.

\section{Summary Box}

\section{What is already known:}

- Because of rising antibiotic resistance, Helicobacter pylori (H. pylori) eradication rate with standard triple therapies is unacceptably low $(<80 \%)$ in most countries

- Hybrid therapy is an innovative 14-day, twostep, non-bismuth quadruple regimen, which functionally combines the concomitant and sequential treatments

- To date, only a limited number of studies have evaluated hybrid therapy in a high-resistance setting, and even fewer provided pretreatment susceptibility data

\section{What the new findings are:}

- This is the largest, to date, European series of antibiotic-resistant $H$. pylori strains treated with hybrid therapy

- Hybrid therapy is an effective (PP cure rate: 90.2\%) and safe first-line regimen for $H$. pylori eradication in populations with relatively high antibiotic resistance rates

- Dual clarithromycin and metronidazole resistance plays a key role in the treatment failure of hybrid therapy 


\section{References}

1. Papastergiou V, Georgopoulos SD, Karatapanis S. Treatment of Helicobacter pylori infection: meeting the challenge of antimicrobial resistance. World J Gastroenterol 2014;20:9898-9911.

2. Georgopoulos SD, Papastergiou V, Karatapanis S. Current options for the treatment of Helicobacter pylori. Expert Opin Pharmacother 2013;14:211-223.

3. Papastergiou V, Georgopoulos SD, Karatapanis S. Treatment of Helicobacter pylori infection: past, present and future. World $J$ Gastrointest Pathophysiol 2014;5:392-399.

4. Georgopoulos SD, Papastergiou V, Karatapanis S. Helicobacter pylori eradication therapies in the era of increasing antibiotic resistance: a paradigm shift to improved efficacy. Gastroenterol Res Pract 2012;2012:757926.

5. Georgopoulos SD, Xirouchakis E, Martinez-Gonzales B, et al. Randomized clinical trial comparing ten day concomitant and sequential therapies for Helicobacter pylori eradication in a high clarithromycin resistance area. Eur J Intern Med 2016;32:84-90.

6. Karamanolis GP, Daikos GL, Xouris D, Goukos D, Delladetsima I, Ladas SD. The evolution of Helicobacter pylori antibiotics resistance over 10 years in Greece. Digestion 2014;90:229-231.

7. Georgopoulos SD, Xirouchakis E, Martinez-Gonzalez B, et al. Clinical evaluation of a ten-day regimen with esomeprazole, metronidazole, amoxicillin, and clarithromycin for the eradication of Helicobacter pylori in a high clarithromycin resistance area. Helicobacter 2013;18:459-467.

8. Malfertheiner P, Megraud F, O'Morain CA, et al; European Helicobacter and Microbiota Study Group and Consensus panel. Management of Helicobacter pylori infection-the Maastricht V/ Florence Consensus Report. Gut 2017;66:6-30.

9. Georgopoulos SD, Xirouchakis E. Which regimens should be used and which rejected for the treatment of Helicobacter pylori? Am J Gastroenterol 2017;112:1168-1169.

10. Hsu PI, Wu DC, Wu JY, Graham DY. Modified sequential Helicobacter pylori therapy: proton pump inhibitor and amoxicillin for 14 days with clarithromycin and metronidazole added as a quadruple (hybrid) therapy for the final 7 days. Helicobacter 2011;16:139-145.

11. Liatsos C, Georgopoulos SD. Helicobacter pylori best treatment approach: should a national consensus be the best consensus? Ann Gastroenterol 2017;30:704-706.

12. Song ZQ, Liu J, Zhou LY. Hybrid therapy regimen for Helicobacter pylori eradication. Chin Med J (Engl) 2016;129:992-999.

13. Wu JY, Hsu PI, Wu DC, Graham DY, Wang WM. Feasibility of shortening 14-day hybrid therapy while maintaining an excellent Helicobacter pylori eradication rate. Helicobacter 2014;19:207-213.

14. Zhou L, Zhang J, Chen M, et al. A comparative study of sequential therapy and standard triple therapy for Helicobacter pylori infection: a randomized multicenter trial. Am J Gastroenterol 2014; 109:535-541.

15. Georgopoulos SD, Xirouchakis E, Mentis A. Is there a nonbismuth quadruple therapy that can reliably overcome bacterial resistance? Gastroenterology 2013;145:1496-1497.

16. Graham DY, Lee YC, Wu MS. Rational Helicobacter pylori therapy: evidence-based medicine rather than medicine-based evidence. Clin Gastroenterol Hepatol 2014;12:177-186.e3.

17. Georgopoulos S, Papastergiou V, Xirouchakis E, et al. Nonbismuth quadruple "concomitant" therapy versus standard triple therapy, both of the duration of 10 days, for first-line $H$. pylori eradication: a randomized trial. J Clin Gastroenterol 2013;47:228-232.

18. Georgopoulos S, Papastergiou V, Xirouchakis E, et al. Evaluation of a four-drug, three-antibiotic, nonbismuth-containing "concomitant" therapy as first-line Helicobacter pylori eradication regimen in Greece. Helicobacter 2012;17:49-53.

19. Metanat HA, Valizadeh SM, Fakheri H, et al. Comparison between 10- and 14-day hybrid regimens for Helicobacter pylori eradication: a randomized clinical trial. Helicobacter 2015;20:299-304.

20. Hsu PI, Wu DC, Wu JY, Graham DY. Is there a benefit to extending the duration of Helicobacter pylori sequential therapy to 14 days? Helicobacter 2011;16:146-152.

21. Song Z, Zhou L, Zhang J, He L, Bai P, Xue Y. Hybrid therapy as firstline regimen for Helicobacter pylori eradication in populations with high antibiotic resistance rates. Helicobacter 2016;21:382-388.

22. Molina-Infante J, Romano M, Fernandez-Bermejo M, et al. Optimized nonbismuth quadruple therapies cure most patients with Helicobacter pylori infection in populations with high rates of antibiotic resistance. Gastroenterology 2013;145:121-128.

23. Georgopoulos SD, Papastergiou V, Karatapanis S. Treatment of Helicobacter pylori infection: optimization strategies in a high resistance era. Expert Opin Pharmacother 2015;16:2307-2317.

24. Bertilsson L, Lou YQ, Du YL, et al. Pronounced differences between native Chinese and Swedish populations in the polymorphic hydroxylations of debrisoquin and S-mephenytoin. Clin Pharmacol Ther 1992;51:388-397.

25. Webber MA, Piddock LJ. The importance of efflux pumps in bacterial antibiotic resistance. I Antimicrob Chemother 2003;51:9-11.

26. Zullo A, Rinaldi V, Winn S, et al. A new highly effective shortterm therapy schedule for Helicobacter pylori eradication. Aliment Pharmacol Ther 2000;14:715-718.

27. Hsu PI, Kao SS, Wu DC, et al; Taiwan Acid-Related Disease Study Group. A randomized controlled study comparing reverse hybrid therapy and standard triple therapy for Helicobacter pylori infection. Medicine (Baltimore) 2015;94:e2104.

28. Tsay FW, Wu DC, Kao SS, et al. Reverse sequential therapy achieves a similar eradication rate as standard sequential therapy for Helicobacter pylori eradication: a randomized controlled trial. Helicobacter 2015;20:71-77.

29. Cuadrado-Lavín A, Salcines-Caviedes JR, Diaz-Perez A, et al. First-line eradication rates comparing two shortened non-bismuth quadruple regimens against Helicobacter pylori: an open-label, randomized, multicentre clinical trial. J Antimicrob Chemother 2015;70:2376-2381.

30. De Francesco V, Hassan C, Ridola L, Giorgio F, Ierardi E, Zullo A. Sequential, concomitant and hybrid first-line therapies for Helicobacter pylori eradication: a prospective randomized study. J Med Microbiol 2014;63:748-752.

31. Heo J, Jeon SW, Jung JT, et al; Daegu-Gyeongbuk Gastrointestinal Study Group. Concomitant and hybrid therapy for Helicobacter pylori infection: A randomized clinical trial. J Gastroenterol Hepatol 2015;30:1361-1366.

32. Oh DH, Lee DH, Kang KK, et al. Efficacy of hybrid therapy as first-line regimen for Helicobacter pylori infection compared with sequential therapy. J Gastroenterol Hepatol 2014;29:1171-1176.

33. Sardarian H, Fakheri H, Hosseini V, Taghvaei T, Maleki I, Mokhtare M. Comparison of hybrid and sequential therapies for Helicobacter pylori eradication in Iran: a prospective randomized trial. Helicobacter 2013;18:129-134.

34. Song ZQ, Zhou LY. Hybrid, sequential and concomitant therapies for Helicobacter pylori eradication: A systematic review and metaanalysis. World J Gastroenterol 2016;22:4766-4775.

35. Tang HL, Li Y, Hu YF, Xie HG, Zhai SD. Effects of CYP2C19 lossof-function variants on the eradication of $H$. pylori infection in patients treated with proton pump inhibitor-based triple therapy regimens: a meta-analysis of randomized clinical trials. PLoS One 2013;8:e62162. 